with ADRF we found that it was independent ( $p$ : 0.002) of the result of capillaroscopy, being the risk of developing the disease in a patient with positive ANA 8.5 times higher than in an ANA negative patient.

Conclusions Capillaroscopy in patients with RP has a high PNV, which allows us to estimated, with high reliability, the association of this phenomenon with autoimmune disease in patients with normal capillaroscopic patterns.

Disclosure of Interest None declared.

\section{P049 AGE-ASSOCIATED B CELLS IN EARLY DRUG-NAÏVE RHEUMATOID ARTHRITIS PATIENTS}

${ }^{1} G$ Vidal Pedrola*, ${ }^{1} \mathrm{~A}$ Pratt, ${ }^{1} \mathrm{~A}$ Mellor, ${ }^{2} \mathrm{D}$ Scheel-Toellner, ${ }^{1} \mathrm{~J}$ Isaacs, ${ }^{1} \mathrm{~A}$ Anderson. 'Inflammation, Immunology and Immunotherapy - William Leech Building, Newcastle University, Newcastle upon Tyne; ${ }^{2}$ Centre for Translational Inflammation Research, University of Birmingham, Birmingham, UK

\subsection{6/annrheumdis-2018-EWRR2019.41}

Career situation of first and presenting author Student for a master or a $\mathrm{PhD}$.

Introduction Rheumatoid arthritis (RA) is a chronic autoimmune disorder characterised by joint inflammation and bone destruction. The presence of autoantibodies, years before the clinical onset of disease, and the efficacy of Rituximab, a Bcell depleting therapy, highlight a pathogenic role for B cells. Different groups have recently identified a novel subset of $\mathrm{B}$ cells named age-associated B cells (ABCs). Studies in mice autoimmune models and patients suffering from autoimmune diseases described these cells as CD $19^{\text {high }} \mathrm{CD} 21^{-} \mathrm{CD} 11 \mathrm{c}^{+}$. Moreover, a subset of synovial fluid B cells with low levels of CD21, expresses FcRL4 and produces the cytokine RANKL, which stimulates the differentiation and activation of osteoclasts. The ABCs found in peripheral blood could therefore be the precursors of this FcRL4 positive subset found in synovia. Objectives We aimed to investigate the proportion and phenotype of peripheral blood $\mathrm{ABCs}$ in patients suffering from early drug naïve RA.

Methods Newly presenting patients, naïve to immunomodulatory treatment, were recruited from the Newcastle Early Arthritis Clinic, and followed until diagnoses were confirmed. B-cell subsets in peripheral blood were detected and phenotyped using flow cytometry. NanoString nCounter Immunlogy v2 Panel (NanoString Technologies) was used to detect mRNA from cell lysates of sorted ABCs, naïve, memory and CD5+ B cells from RA patients, age-matched healthy controls and disease controls (Psoriatic Arthritis patients).

Results Our work showed that there are no significant differences in the frequency of ABCs between RA patients, disease controls and age-matched healthy controls. There is a possible trend for increased frequencies of ABCs with age as well as those who have a high disease activity. Our results also show that $\mathrm{ABCs}$ resemble a memory $\mathrm{B}$ cells phenotype with regard to class-switch immunoglobulins expression, with a significant percentage of them being positive for $\operatorname{IgG}$ and $\operatorname{IgA}$. Interestingly, the $\mathrm{FcRL}_{4}^{+}$, the proliferating $\mathrm{Ki}^{+} 7^{+}$and the T-bet expressing $\mathrm{B}$ cells were enriched in the $\mathrm{ABC}$ population compared to the other $\mathrm{B}$ cell subsets. Furthermore, ABCs expressed high levels of MHC class II and co-stimulatory molecules, as well as the activation marker, CD69. In addition, gene expression analysis using NanoString showed differentially expressed genes between $\mathrm{ABCs}$ and memory B cells.
Conclusions These data supports an activated phenotype of the ABCs, which supports the idea that ABCs have a pathogenic role in RA, potentially via autoantibody and $\mathrm{T}$ cell stimulatory ability. However, further characterisation of this subset and functional studies are needed.

Disclosure of Interest None declared.

\section{P050 JAN GÖSTA WALDENSTRÖM AND RHEUMATOLOGY}

F Wollheim*. Department of Clinical Sciences Lund, Rheumatology. Medical Faculty, Lund University, Lund, Sweden

\subsection{6/annrheumdis-2018-EWRR2019.42}

Career situation of first and presenting author Post-doctoral fellow.

Introduction On 2. September 1943 Jan Waldenström (19061996) successfully submitted a paper to Acta Medica Scandinavica describing two patients with a new disease. The discovery was to make him world famous. ${ }^{1}$ This year marks the 75 th anniversary of macroglobulinemia and it coincided with the 10th biennial international workshop of Waldenström's macroglobulinemia, discussing advances in the genetic basis, pathogenesis and treatments of the disease. ${ }^{2}$ Jan Waldenström 7[MO1] (JW) would have enjoyed this workshop immensely, sharing the information that over $95 \%$ of the patients had somatic mutations affecting the MYD88 gene on the 2nd chromosome as well as the impressive advances in treatment. Attending this excellent meeting brought back memories of my time as Waldenström's $\mathrm{PhD}$ student and triggers me to compose this vignette, focusing on connections between the interests of my mentor and rheumatology. For a more comprehensive account of Jan Waldenström's legacy I recommend Robert Kyle's superb obituary, published in Blood, a journal JW was attached to from its start. ${ }^{3}$

After the successful defence of his landmark PhD Thesis on acute intermittent porphyria ${ }^{4}$ Waldenström's interest focused on haematology. He worked in Uppsala, a university where the study of proteins had prominence. There The Svedberg had developed the ultracentrifuge and Arne Tiselius the free electrophoresis and Robin Fåhreus the ESR. Conditions with elevated sedimentation rate (ESR) caught his special attention. An early witness is a paper from 1937, analysing 5 cases diagnosed with uveoparotitis, a rare condition most prevalent in women and considered by many to be a form of tuberculosis. He noticed the several multiorgan manifestations, the similarities with von Mikulits' disease and Boeck's sarcoid, the presence of high ESR, unspecific Wasserman reaction, absence of proof of tuberculosis, presence of xerostomia, and frequent CNS manifestations. ${ }^{5}$ Today the diagnosis could have been IgG4 related disease in several of these patients. This early paper also shows a keen interest in inflammatory systemic conditions.

In the early $1940 \mathrm{~s}$ JW collected serum from some 100 patients with longstanding ESR exceeding $120 \mathrm{~mm}$, and had it analysed by the new technique of free boundary electrophoresis by K.O. Pedersen in the Department of Physical Chemistry. In 1943 he described 3 cases from this population characterised by repeated bouts of declive purpura, leaving spots of brown discoloration, mild anaemia, and on the whole, good general health. Two of the 3 women also had dry eye problems and one had dry mouth and swollen parotis glands. $\mathrm{He}$ named the condition 'purpura 
hyperglobulinemica'. ${ }^{6}$ Similar patients were soon identified by others and labelled 'Waldenström's purpura hyperglobulinemica', citing his Swedish communication that contained only a brief summary in English. A more comprehensive later report, presented new cases, detailed case histories and a colour illustration of the typical skin changes, figure 1 , and discussed the systemic nature of the condition in depth. Other organ manifestations included lymphadenopathy, uveoparotitis, Sjögren's syndrome and SLE. The serum albumin concentration remained normal in line with the benign nature of the condition. ${ }^{7}$ The author was surprised that this English paper was hardly ever cited. ${ }^{8}$

In 1949 Jan Waldenström was appointed as the first professor and chairman of internal medicine at Malmö General Hospital, the new second teaching hospital of the Lund University. In Malmö he continued investigating what he now called gammapathies. The new technique of paper electrophoresis refined by Carl-Bertil Laurell dramatically simplified identification of patients with hypogammaglobinaemia and serum electrophoresis became a routine test. In collaboration with Sten Winblad sera were also routinely examined for presence of antibodies to bacterial antigens by a package called 'total serology'. Combined, this lead to the distinction between polyclonal reactive and monoclonal malignant conditions, perhaps JW's most important scientific contribution. ${ }^{9}$

In $1950 \mathrm{JW}$ was invited to speak at the first German congress of Gastroenterology in Germany after the war. There he presented a few cases of a new form of active chronic hepatitis, predominantly in young women with high ESR, very high concentration of gamma-globulin and prominence of plasma cells in the liver. Some but not all developed cirrhosis. ${ }^{10}$ In 1951 a similar observation was presented as an abstract by Henry Kunkel's group. ${ }^{11}$ This condition was also soon observed by other investigators and known under several names. One that has survived is chronic active hepatitis. Sheila Sherlock has summarised the clinical spectrum of the disease based on 115 of her own cases, and emphasised the systemic nature which is characteristic of an autoimmune disorder. ${ }^{12}$ Ulcerative colitis, skin rashes, glomerulonephritis, pulmonary infiltrates and Hashimoto's thyroiditis were common. ANAs were found in $40 \%$ and rheumatoid factor in $70 \%$ of her cases.

In Malmö JW soon emerged as a charismatic leader, equally popular among patients, medical students, staff, and highly respected by Malmö's ambitious administrators. Within few years the department, although frugally staffed, became the leading academic internal medicine unit in the country. JW was a firm believer in the blessing of un-fragmented internal medicine, although expecting members of the staff to select an area of special expertise within it. Rheumatology was only established as a speciality in Sweden in 1969 and the first generation of rheumatologists were specialists in internal medicine. But in Malmö autoimmune disorders like SLE were specilalité de la maison and Talbott and Ferrandis' 'Collagen Diseases' was obligatory reading. ${ }^{13}$ The book from 1956 still rests on my shelf.

International visitors were frequent guests and fellows came to work with the famous professor. Patients with rare or unclear disease were referred to him from all over Sweden. One example which directly affected me as the most junior house officer was two cases with frequent infections, antibody deficiency disease, labelled as adult 'acquired' hypogammaglobinaemia. The professor had interviewed the women who came from different hospitals in the country and not simultaneously. In spending good time talking to them he happened to find out that both had roots in a village 100 miles to the north of Malmö. I was given the task to find out if they had common ancestors. After some months of searching in old church registers this in fact turned out to be the case, hinting at a possible genetic etiology. When I showed the pedigree starting in the mid eighteens century to the professor, JW was of course pleased, and when I presented the brief report with his name after mine he said 'Fine, send it to The Lancet'. Unfortunately he erased his own name from the manuscript although it clearly was his idea based on his ground work. The paper was accepted without changes. ${ }^{14}$ The story was later supported by a following larger report. ${ }^{15}$ The disease now is named common variable immunodeficiency and genomic technology including next-generation sequencing reveals its complex genetic basis and explains links to autoimmunity. ${ }^{16}$ The Lancet paper was my first publication as internist and it opened the way to USA. Just coming from JWs department in Malmö usually resulted in red carpet reception.

Several of JW's international contacts and visitors were prominent in rheumatology. Henry Kunkel, Morris Ziff, Eric Bywaters, Barbra Ansell, Norman Talal, Eng Tan, Bob Winchester and Ralph C Williams are some names that come to mind (figure 2). He never passed New York without visiting Henry Kunkel at the Rockefeller Institute where he enjoyed making ward rounds. A paper titled 'Forty years with the gammaglobulins' ${ }^{17}$ gives further personal proof of JW's close ties with rheumatology, which certainly facilitated my path into the specialty. My own visits with Henry Kunkel's small group usually included a seminar where the presenter was allowed to use the blackboard but not to show slides. The group then had lunch and after lunch went to the library and browsed through the new journals of the day. Electronic journals had not been born.

\section{REFERENCES}

1. Acta med Scand 1944;117:216-247.

2. 10th International Workshop on Waldenström's Macroglobulinemia [WM] New York Marriott Downtown, New York, NY.

3. Acta Med Scand 1937;suppl 82:1-254.

7. Waldenström J. Three new cases of purpura hyperglobulinemica. A study in longlasting benign increase in serum globulin. Acta Med Scand Suppl. 1952;266:931-46.

10. Waldenström J. Leber, Blutproteine und Nahrungseiweiss. Dtsch Z Verdau Stoffwechselkr 1953;9:113-9.

11. Kunkel HG, Ahrens Jr EH, Eigenmenger WJ, Bonngiovanni AM, Slater RJ. Extreme hypergammaglobulinemia in young women with liver disease of unknown etiology. J Clin Invest 1951;30:654.

12. Sherlock S. Waldenström's chronic active hepatitis. Acta Med Scand Suppl. 1966;445:426-33

13. Talbott RM, Ferrandis JH. Collagen diseases. Grune and Stratton, New York and London 1956;1-232.

14. Wollheim F. Inherited 'acquired' hypogammaglobulinaemia. Lancet 1961 February 11;1(7172):316-7

15. Wollheim FA, Belfrage $S$, Coester $C$, Lindholm H. Primary 'acquired' hypogammaglobulinemia; clinical and genetic aspects of nine cases. Acta Med Scand 1964 July;176:1-16.

16. Kienzler AK, Hargreaves CE, Patel SY. The role of genomics in common variable immunodeficiency disorders. Clin Exp Immunol 2017;188(3):326-332.

17. Waldenström JG. Forty years with the gammaglobulins. Scand I Immunol $1987: 25: 211-218$ 
Disclosure of Interest None declared.

\section{P051/001 CHARACTERIZATION OF THE ANTI-CENTROMERE ANTIBODY RESPONSE IN SYSTEMIC SCLEROSIS PATIENTS SUGGESTS A BROAD AND ACTIVE B CELL RESPONSE}

${ }^{1} \mathrm{CM}$ Wortel ${ }^{*},{ }^{1} \mathrm{NM}$ van Leeuwen, ${ }^{1} \mathrm{M}$ Boonstra, ${ }^{2} \mathrm{JA}$ Bakker, ${ }^{1} \mathrm{RE}$ Toes, ${ }^{1} \mathrm{TW}$ Huizinga, ${ }^{1} \mathrm{JK}$ de Vries-Bouwstra, ${ }^{1} \mathrm{HU}$ Scherer. ${ }^{1}$ Rheumatology; ${ }^{2}$ Clinical Chemistry and Laboratory Medicine, Leiden University Medical Center, Leiden, Netherlands

\subsection{6/annrheumdis-2018-EWRR2019.43}

Career situation of first and presenting author Student for a master or a $\mathrm{PhD}$.

Introduction Systemic Sclerosis (SSc) is a rare, heterogeneous autoimmune disease characterized by microvascular damage, organ fibrosis and immune dysfunction. Autoantibodies are detected in $>95 \%$ of patients, the most prevalent being anticentromere (ACA) and anti-topoisomerase (ATA) antibodies. Although used for diagnosis, little is known about the underlying auto-reactive B cell responses. In particular, the ACA B cell response has been poorly studied.

Objectives Characterization of the ACA B cell response in SSc patients.

Methods ACA IgG, IgA and IgM levels were measured in serum samples of 167 ACA IgG + SSc patients. Patients were divided in a SSc (fulfilling ACR 2013 criteria, $n=132$ ) and a very early SSc group (fulfilling VEDOSS criteria, $n=35$ ). Additionally, PBMCs from ACA IgG+ SSc patients (and ATA IgG + SSc and healthy donors (HD) as control) were cultured either in the presence of CD40L expressing fibroblasts, IL-21 and BAFF or without stimulation. Levels of ACA IgG, IgA and IgM (and total Ig) were measured after one week of culture using ELISA.

Results ACA IgG+ SSc patients displayed a broad isotype usage with $75 \%$ being ACA IgA+ and $68 \%$ being ACA IgM + in serum. Patients within the SSc group showed higher ACA IgG levels and a higher percentage of ACA IgM positivity compared to the very early SSC group. ACA IgG, IgA and IgM could be measured in ACA SSc PBMC culture medium following stimulation, but not in ATA SSc and HD, indicating the presence of circulating ACA B cells of all three isotypes. In cultures that yielded sufficient Ig production, ACA IgG was detectable in 7/9 ACA SSc patients, ACA IgA in $3 / 7$ and ACA IgM in $2 / 7$. Furthermore, ACA IgG production was also detected in the absence of stimulation in 5/9 patients, suggesting the presence of ACA-producing plasmablasts in the circulation. No spontaneous production of antiTetanus Toxoid antibodies, a control recall response, was observed.

Conclusions ACA + SSc patients display a broad range of isotype usage in their ACA response, reflected both by ACA serum levels and presence of ACA IgG-, IgA- and ACA IgM-producing B cells in the peripheral blood. Additionally, ACA IgG production by unstimulated PBMCs points towards continuous differentiation of memory cells into antibody secreting cells. These data, together with differential isotype profiles between very early SSc and SSc patients, provide insight into the ACA B cell response and its potential involvement in disease-relevant pathogenetic processes.

Disclosure of Interest None declared.

\section{P052 AUTO-ANTIBODIES AGAINST POST TRANSLATIONAL MODIFIED PROTEINS IN OSTEOARTHRITIS: PILOT DATA COMPARING SYNOVIAL FLUID AND SERA}

${ }^{1} \mathrm{X} X \mathrm{Xie}{ }^{*},{ }^{2} \mathrm{MV}$ Delft, ${ }^{2} \mathrm{~L}$ Trouw, ${ }^{1} \mathrm{P}$ Conaghan, ${ }^{1} \mathrm{~F}$ Ponchel. ' ${ }^{2}$ Leeds Institute of Rheumatic and Musculoskeletal Medicine, The University of Leeds, Leeds, UK; ${ }^{2}$ Leiden University Medical Centre, Leiden University, Leiden, Netherlands

\subsection{6/annrheumdis-2018-EWRR2019.44}

Career situation of first and presenting author Student for a master or a $\mathrm{PhD}$.

Introduction Osteoarthritis (OA) is a highly prevalent disease and a leading cause of disability worldwide. OA is age-related and as such has a potential devastating impact on our ageing populations. The pathogenesis of $\mathrm{OA}$ remains poorly understood. Inflammatory responses are prevalent in driving processes associated with aging, and increased levels of IgG autoantibodies (auto-Abs) have been associated with age. In $\mathrm{OA}$, auto-Abs are also frequently observed, notably towards post translational modified (PTM) proteins. Such PTM are often the result of inflammation-driven mechanisms such as oxidative stress, carbamylation and citrullination ${ }^{1}$ in $\mathrm{OA}$ joints. Objectives To establish a profile of auto-Abs associated with PTM in OA and controls.

Methods Serum was collected from healthy controls (HC) and OA patients as well as rheumatoid arthritis (RA); knee synovial fluid (SF) samples were collected from OA/RA patients. All participants gave informed consent. The levels of antibodies against citrullinated protein antigens (ACPA) was measured (Phadia CCP-test), antibodies against carbamylated proteins (anti-CarP) were measure in collaboration with Leiden using an in-house ELISA, ${ }^{2}$ antibodies against glycated-collagen proteins (anti-ROS-CII) were analysed using an in house ELISA. ${ }^{3}$

Results ACPA positivity was present in 6\% $(n=24 / 392)$ of OA patients' serum, which was 3 times more frequent that in $\mathrm{HC}$ $(\sim 2 \%)$, but was present in $48 \%(n=22 / 46)$ of OA SF. RA frequencies were $73 \%(n=41 / 56)$ in SF and 65\% $(n=55 / 85)$ in serum. Anti-CarP auto-antibodies were detected in $11 \%(n=12 /$ $111)$ of OA sera and in $37 \%(n=127 / 340)$ of RA sera (SF results are not yet available). Anti-collagen auto-Abs in serum were observed at similar frequencies: $18 \%$ of OA $(n=6 / 34)$ and $18 \%(n=14 / 78)$ of RA patients, but only $6.7 \%(n=3 / 45)$ of OA fluids and $3.6 \%(n=2 / 56)$ of RA fluids were positive. Collagen modified by reactive oxygen species enables detection of auto-Ab (anti-ROS-CII); these were observed in 35\% $(n=12 / 34)$ of OA and $32 \%(n=25 / 78)$ of RA sera; this differed from SF with $20 \%$ positive $(n=9 / 45)$ in OA and $60 \%(n=34 / 56)$ in RA. Conclusions In $\mathrm{OA}$ patients, autoimmune responses against native proteins (especially collagens and proteoglycans) have been reported since the 1980 's, notably in relation with severity. ${ }^{4}$ However, our data demonstrate that auto-Abs may also result from the accumulation of PTMs induced by chemical reactions in the inflamed joint. These auto-Abs against PTM-antigens may also be contributing to disease severity, as these are notably frequently detected in SF even if a bit less frequently than in RA in our pilot cohorts.

\section{REFERENCES}

1. Burska AN. MI 2014;492873-492873.

2. Shi J, et al. ART 2015:17:339

3. Strollo $R$, et al. A\&R 2013;65:1702-12.

4. Yuan GH, et al. A\&R 2003;48:602-611.

Disclosure of Interest None declared 